\title{
Heart Rate Recovery in Decision Support for High Performance Athlete Training Schedules
}

\author{
David J. Cornforth \\ Applied Informatics Research Group, University of Newcastle, \\ Callaghan, NSW, Australia \\ David.Cornforth@Newcastle.edu.au
}

Dean J. Robinson

Corio Bay Sports Medicine Centre, Geelong, VIC, Australia

dj.robinson09@gmail.com

\author{
lan Spence \\ Discipline of Pharmacology, Faculty of Medicine, \\ Sydney University, Sydney, Australia
}

ian.spence@sydney.edu.au

Herbert F. Jelinek

Centre for Research in Complex Systems and School of

Community Health, Charles Sturt University, Albury, Australia, and Australian School of Advanced Medicine, Macquarie University, Sydney, Australia

\section{HJelinek@csu.edu.au}

\begin{abstract}
Material published as part of this publication, either on-line or in print, is copyrighted by the Informing Science Institute. Permission to make digital or paper copy of part or all of these works for personal or classroom use is granted without fee provided that the copies are not made or distributed for profit or commercial advantage AND that copies 1) bear this notice in full and 2) give the full citation on the first page. It is permissible to abstract these works so long as credit is given. To copy in all other cases or to republish or to post on a server or to redistribute to lists requires specific permission and payment of a fee. Contact Publisher@InformingScience.org to request redistribution permission.
\end{abstract}

This work investigated the suitability of a new tool for decision support in training programs of high performance athletes. The aim of this study was to find a reliable and robust measure of the fitness of an athlete for use as a tool for adjusting training schedules. We examined the use of heart rate recovery percentage ( $\mathrm{HRr} \%)$ for this purpose, using a two-phased approach. Phase 1 consisted of testing the suitability of $\mathrm{HRr} \%$ as a measure of aerobic fitness, using a modified run-

ning test specifically designed for highperformance team running sports such as football. Phase 2 was conducted over a 12-week training program with two different training loads. $\mathrm{HRr} \%$ measured aerobic fitness and a running time-trial measured performance. Consecutive measures of $\mathrm{HRr} \%$ during phase 1 indicated a Pearson's $r$ of 0.92 , suggesting a robust measure of aerobic fitness. Dur- 
ing phase $2, \mathrm{HRr} \%$ reflected the training load and significantly increased when the training load was reduced between weeks 4 to 5. This work shows that $\mathrm{HRr} \%$ is a robust indicator of aerobic fitness and provides an on-the-spot index that is useful for training load adjustment of eliteperformance athletes.

Keywords: heart rate recovery, decision support, training schedule, high performance sport, aerobic fitness

\section{Introduction}

The aim of this study was to determine whether heart rate recovery percentage ( $\mathrm{HRr} \%)$ is a robust measure of athlete fitness over time, and whether $\mathrm{HRr} \%$ changes reflect changes in fitness, performance, and training load over a 12-week training program, in the context of a professional team sport environment. The training environment in this study closely reflects actual on-field conditions of the athletes. Results show that $\mathrm{HRr} \%$ is a robust indicator of aerobic fitness and match performance. Also, $\mathrm{HRr} \%$ provides an on-the-spot index that is useful for making adjustments to the training load of elite-performance athletes.

A successful pre-season training program aims to increase the fitness of athletes in preparation for the upcoming competition season. This is achieved through the application of training protocols that are similar to those encountered in performance situations during the competitive season. These stressful conditions may take a number of different formats from traditional running to game-based conditioning drills. The training program for elite athletes needs to allow each athlete to reach peak performance at the appropriate time and to maintain this level of performance throughout the competition season. At the same time, the training program must avoid overtraining and fatigue by controlling the total amount of exercise, or load. Athletes have a finite capacity for adaptation to training load before fatigue and over-reaching occurs (Coutts, Reaburn, Piva, \& Rowsell, 2007). Fatigue and over-reaching during training can limit the athlete's performance capacity and may take several weeks to recover from (Kreider, Fry, \& O'Toole, 1998). The ability to monitor and adjust athletes' training load according to their training status and/or their health status is therefore essential. An appropriate and sensitive measure allows for an optimal training load to be applied and over-training to be avoided.

Any measurement tool used to determine an athlete's training and health status must be accurate, reliable, and able to determine real change appropriate for high-performance athletes. Several models have addressed how to determine the correct level of training to obtain the best performance outcomes and how these can be monitored (Busso, 2003). Some of these monitoring tools attempt to measure the overall well-being of the athlete using questionnaire data, such as the Profile of Mood Status (POMS) questionnaire (McNair, Lorr, \& Droppleman, 1971), the Daily Analysis of Life Demands for Athletes Questionnaire (DALDA) (Rushall, 1990) and Kenttä's passive and/or active recovery scale (Kenttä \& Hassmen, 1998).

\section{Autonomic Nervous System Assessment in Athlete Performance}

An alternative way of monitoring training status is to analyze the response of the autonomic nervous system to training load. A variety of measurement tools for monitoring and predicting changes in training status have been developed (Borresen \& Lambert, 2008; Lamberts, Rietjens, Tijdink, Noakes, \& Lambert, 2010; Lamberts, Swart, Capostagno, Noakes, \& Lambert, 2009). A period of submaximal exercise has been shown to be associated with a prolonged period of increased heart rate as a function of increased sympathetic activity extending up to 45 minutes post exercise with parasympathetic reactivation peaking at two minutes post exercise (Wang et al., 2011). Heart Rate recovery (HRr) is defined as the fall in heart rate over a given period following 
exercise. $\mathrm{HRr}$ is strongly correlated to parasympathetic activity early in the recovery period, and thus autonomic regulation of the heart rate can provide important information on athlete psychological stress, fatigue, fitness, and over-stretching (Kannankeril, Le, Kadish, \& Goldberger, 2004; Savin, Davidson, \& Haskell, 1982). Several studies have reported autonomic nervous system changes in association with training performance outcomes using $\mathrm{HRr}$ as a measure of autonomic response (Borresen \& Lambert, 2008; Hottenrott, Hoos, \& Esperer, 2006; Lamberts et al., 2009). In other studies, a decrease in submaximal exercise heart rate (SubHR) (Barbeau, Serresse, \& Boulay, 1993; Scharhag-Rosenberger, Meyer, Walitzek, \& Kindermann, 2009), quicker HRr (Buchheit et al., 2008; Sugawara, Murakami, Maed, Kuno, \& Matsuda, 2001) and greater postexercise heart rate variability (HRV) (Buchheit, Duche, Laursen, \& Ratel, 2010) have been related to changes in aerobic fitness as a measure of performance.

The usefulness of a marker to assess physiological adaptation to training ideally requires it to be easy to administer so that frequent monitoring is possible with little inconvenience to the athlete (Borresen \& Lambert, 2008). From the literature, we can identify suitable candidates such as SubHR (Buchheit et al., 2008; Lamberts, Lemmink, Durandt, \& Lambert, 2004; Lamberts et al., 2009), post-exercise HRr (Bosquet, Gamelin, \& Berthoin, 2008; Buchheit et al., 2008; Lamberts et al., 2004) and HRV (Buchheit et al., 2008; Buchheit, Papelier, Laursen, \&Ahmaidi, 2007; Javorka, Zila, Balharek, \& Javorka, 2002; Martinmäki \& Rusko, 2007). The response of the cardiac autonomic nervous system can be assessed non-invasively using these measures, all of which can provide useful information regarding the functional adaptations to a given training stimulus. The reliability and the associated error of measurement of these markers have been previously described mainly in individual sports such as cycling and swimming using treadmill or bicycle programs for performance and aerobic fitness testing.

The utilization of the warm-up period to collect submaximal heart rate (SubHR), post-exercise $\mathrm{HRr}$ and/or HRV recordings minimizes the disturbance to the athletes (Buchheit et al., 2010). To be able to adapt training loads based on the measurements within the warm-up requires confidence with the results. Therefore the 'normal' day-to-day variations of these indices need to be taken into account (Hopkins \& Hewson, 2001). Heart rate recovery is a robust indicator of cardiac function and measures of $\mathrm{HRr}$ rather than of HRV avoid saturation effects and allow assessment of exercise outcomes (Kiviniemi et al., 2004). In individual high-performance sport, HRr is used to gauge aerobic fitness and the effect of training. Fitter athletes may have a faster HRr, with HRr increasing with VO2max (Aziz, Kilding, \&Teh, 2006; Lamberts et al., 2004).

Previous studies on athletes from team sports utilizing a variety of measures have produced conflicting results in part due to a wide variation in baseline fitness of athletes and exercise protocols (Buchheit, Simpson, Al Haddad, Bourdon, \& Mendez-Villanueva, 2012; Edwards, MacFayden, \& Clark, 2003; Hoffman, Epstein, Einbinder, \& Weinstein, 1999). These studies also differed with respect to the time at which $\mathrm{HRr}$ was measured and whether $\mathrm{HRr}$ was measured following sub-maximal or maximal exercise exertion. No systematic study has addressed the reliability of $\mathrm{HRr}$ nor investigated the relationship between $\mathrm{HRr}$ and training load and fatigue in highperformance athletes during a pre-season training regimen. $\mathrm{HRr} \%$ has not previously been used to monitor the effect of pre-season training load and compared to performance outcomes of elite athletes at an individual or team level.

$\mathrm{HRr} \%$, used to assess fitness/fatigue in elite athletes, is dependent on various factors such as training load and needs to be considered in conjunction with athlete performance as part of a preseason training program. An optimum balance between training load and recovery, as measured by heart rate parameters, is essential to an effective training schedule and on-field performance (Baumert, Brechtel, Lock, Voss, \& Abbott, 2006; Borresen \& Lambert, 2008; Meeusen, Watson, Hasegawa, Roelands, \& Piacentini, 2006). 
The work reported in this paper is in two phases. The first phase establishes the reliability of the $\mathrm{HRr} \%$ assessment procedure; the second phase used $\mathrm{HRr} \%$ to assess performance and fatigue in a cohort of high-performance athletes during a 12-week pre-season training period with varying load.

\section{Research Objectives}

From the foregoing, we have shown the need for a robust measure of fitness in elite athletes, a measure that will reflect the changes in athlete fitness during training. It is also required that the measure is easy to administer on the field, and that results are quickly obtained and in a meaningful format, so that the team coach can interpret these results and adjust the athletes training program on the spot. As the literature has drawn attention to $\mathrm{HRr} \%$, this measure forms the focus of this study. The objectives of this research are:

1. To find a reliable and robust measure of the fitness of an athlete

2. To test the suitability of $\mathrm{HRr} \%$ as a measure of aerobic fitness

3. To determine whether $\mathrm{HRr} \%$ is robust, that is, whether changes reflect changes in fitness, performance, and training load over a training program

4. To find evidence to support the use of $\mathrm{HRr} \%$ as an on-the-spot index that is useful for training load adjustment of elite-performance athletes

\section{Methods}

Participants in both parts of the study were professional Australian Football players. Before the testing period, all participants completed a medical screening questionnaire and underwent a battery of medical and musculoskeletal tests. This work was carried out in two phases.

\section{Phase 1: Development of the Heart Rate Recovery Test}

The first phase took place during a 4-day period prior to the commencement of the pre-season training program. Athletes underwent two recovery tests separated by three days, one at the beginning, and the other at the end of this period. On each of these two occasions, heart rate measurements were made during the training warm-up period to minimize the disturbance to the athletes. The heart rate recovery test, modified from the submaximal running test, is also known as a HIMS test (Heart rate Interval Monitoring System). This test was designed to be performed frequently in the warm-up of a training session and has to be submaximal and non-aversive for the athletes (Lamberts, Maskell, Borresen, \& Lambert, 2011). The principal change from the original HIMS test made for the purposes of this study was to use continuous running instead of a stopstart protocol. The pace of running within each of the 4 stages $(9.3,11.1,12.8$, and $14.6 \mathrm{~km} / \mathrm{h}$, respectively) was increased from the original HIMS test to retain $85-90 \%$ maximal heart rate as recommended by Lamberts et al. (2009) and Dellal et al. (2010). Running speeds were adjusted based on the fitness of the athletes in the study. The test was also changed to continuous running in order to minimize the stress to the groin region, as seen in a continual stop-start test, with changes in direction of running as in the HIMS protocol.

The heart rate recovery test was conducted at the same time on each day to avoid any confounding effects due to circadian changes in heart rate. The test consisted of four 2-minute periods of running at progressively increasing speeds $(9.3,11.1,12.8$, and $14.6 \mathrm{~km} / \mathrm{h})$ with athletes resting for one minute between each of the running periods (Reilly, Robinson, \& Minors, 1984). The protocol was designed so that athletes achieved $85-90 \%$ of maximum HR at the end of the fourth period. The distance was broken into 50 -metre blocks, each covered in a set time by a pacing runner in order to maintain the correct speed. 
All athletes were familiar with the training ground and surface used for the testing and had a minimum of four repetitions for familiarization to the test procedures. The participants performed a submaximal continuous running test in Indian file formation of 4 stages with duration of 2 minutes each, followed by a rest period of 1 minute. The total duration of the test was 12 minutes. During the three initial rest periods, the participants stretched. At the end of the run athletes remained standing and stationary. Athletes measured their carotid pulse for 15 seconds (HR Stage 4) and again 1 minute later (HR 1 min post) manually as this was more practical than using electronic recording devices linked with GPS systems, and it had the advantage of providing on the spot results. All athletes were trained in the procedure and verified that they all correctly identified the pulse and counted accurately. A 3-second count into sampling the pulse was used at both time points to allow for correct location of the pulse (Chatterjee, Chatterjee, \&Bandyopadhyay, 2005).

The two recordings (HR Stage 4 and HR 1 min post) were used to calculate the heart rate recovery percentage $(\mathrm{HRr} \%)$ using the formula:

$H R r \%=\frac{(\text { HR Stage } \mathbf{4}-\text { HR } \mathbf{1} \min \llbracket \text { Post }) \rrbracket}{\text { HR Stage } \mathbf{4}} \times 100$

The test was conducted twice, three days apart, to test the reliability/robustness of the measurements.

\section{Phase 2: Application of the Heart Rate Recovery Test}

After the 4-day warm up period (phase 1), athletes participated in a minimum of 15 hours per week of training during the twelve-week pre-season study. Athletes undertook a $2 \mathrm{~km}$ time trial on an Athletics Australia approved track utilizing the track photo-finish timing system at the times indicated in Table 1. Within 5 seconds of completing the time trial, the athletes measured their carotid pulse for 15-seconds and heart rate (HR) in beats per minute (bpm) was calculated. This provided heart rate data for weeks $1,3,6$ and 12, and a measure of performance for weeks 1 , 6 and 12.

\begin{tabular}{|l|l|l|l|}
\hline \multicolumn{3}{|l|}{ Table 1. Testing protocol. Note the absence of a time trial in week 3. } \\
\hline Week 1 & Week 3 & Week 6 & Week 12 \\
\hline HRr test & HRr test & HRr test & HRr test \\
\% Body fat & & & \\
Height & & & \\
\hline $2 \mathrm{~km}$ Time Trial & & $2 \mathrm{~km} \mathrm{TT}$ & $2 \mathrm{~km} \mathrm{TT}$ \\
\hline
\end{tabular}

The training schedule consisted of two blocks of heavy training (Table 2) separated by a twoweek block of lighter training (Table 3). The training regimen was based on best practice and by consultation with the high-performance training coach.

On the second day of the trial each player's height was measured with a calibrated Lufkin tape measure, and body mass was obtained via a calibrated scale (Life Measurement Instruments, Concord, CA). Percent body fat determination was obtained using air-displacement plethysmography with the BOD POD body-composition system (Life Measurement Instruments) using the basic methods previously described (Kraemer et al., 2005). On test days, the body mass of the subjects and the ambient temperature and humidity were measured before each test. 


\begin{tabular}{|l|l|l|l|l|l|l|l|}
\hline \multicolumn{7}{|c|}{ Table 2. Heavy training Schedule Week 1-3 and 6-12 } \\
\hline & Monday & Tuesday & Wednesday & $\begin{array}{l}\text { Thursda } \\
\mathrm{y}\end{array}$ & Friday & Saturday & $\begin{array}{l}\text { Sunda } \\
\text { y }\end{array}$ \\
\hline A & $\begin{array}{l}\text { Skill Condi- } \\
\text { tioning }\end{array}$ & $\begin{array}{l}\text { Technica } \\
\text { 1 Skills }\end{array}$ & $\begin{array}{l}\text { Skill Condi- } \\
\text { tioning }\end{array}$ & Day Off & $\begin{array}{l}\text { Skill Condi- } \\
\text { tioning }\end{array}$ & $\begin{array}{l}\text { Conditionin } \\
\text { g }\end{array}$ & $\begin{array}{l}\text { Day } \\
\text { Off }\end{array}$ \\
\cline { 1 - 3 } PM & Weights & $\begin{array}{l}\text { Rest / } \\
\text { Recov- } \\
\text { ery }\end{array}$ & Weights & & Weights & $\begin{array}{l}\text { Rest / Re- } \\
\text { covery }\end{array}$ & \\
\hline
\end{tabular}

\begin{tabular}{|c|c|c|c|c|c|c|c|}
\hline \multicolumn{8}{|c|}{ Table 3. Light training Schedule Week 4 - 5} \\
\hline & Monday & Tuesday & Wednesday & Thursday & Friday & Saturday & Sunday \\
\hline AM & Conditioning & Day Off & Conditioning & Day Off & Conditioning & Day Off & $\begin{array}{l}\text { Day } \\
\text { Off }\end{array}$ \\
\hline PM & Weights & & Weights & & Weights & & \\
\hline
\end{tabular}

The coefficient of variation was calculated as (SD/mean) x 100 . The reliability statistics are expressed as the change in the mean between measurements, the standard error of measurement (typical error), and the test-retest Pearson correlation (r). The 95\% confidence interval (CI) for the heart rate and all associated parameters was determined for stage 4 and the recovery period. The Kolmogorov-Smirnov test was applied to test for the normality and variance of the distribution. The Kruskal-Wallis test was used to compare outcomes for $\mathrm{HRr}$ in phase 1 and $\mathrm{HRr} \%, 2 \mathrm{kmTT}$ and HR in phase 2 of our protocol.

The between-group differences were assessed as standardized mean differences (Cohen's d), which were calculated using the pooled standard deviations of the two testing sessions. Cohen's d interpretation is based on small effect $=0.2$, medium effect $=0.5$ and large effect $=0.8$ (Cohen, 1988). Statistical analysis was performed using SPSS 20 (IBM, NY, USA).

\section{Results}

The average temperature on the trial days was $25.5 \pm 0.7^{0} \mathrm{C}$ with humidity at $85 \pm 7 \%$. Characteristics of the athletes in the two phases of the study are summarized in Table 4. Figures are expressed as mean \pm standard deviation.

\begin{tabular}{|l|l|l|}
\hline \multicolumn{3}{|c|}{ Table 4. Athlete characteristics for the HRr\% trial } \\
\hline & Phase 1 & Phase 2 \\
\hline Number & 20 & 27 \\
\hline Age (yrs) & $20 \pm 3$ & $20 \pm 3$ \\
\hline Body mass (kg) & $87.4 \pm 10$ & $83.9 \pm 9$ \\
\hline Height (cm) & $189 \pm 8$ & $187 \pm 7$ \\
\hline Lean Body mass (kg) & $77.9 \pm 8.8$ & $75.9 \pm 8.2$ \\
\hline Fat mass \% & $10.8 \pm 3$ & $9.3 \pm 3$ \\
\hline Maximum heart rate (bpm) & $197 \pm 12$ & $197 \pm 13$ \\
\hline 2 km time trial (s) & $425 \pm 27$ & $431 \pm 32$ \\
\hline
\end{tabular}




\section{Phase 1: HRr\% for Measurement of Aerobic Fitness}

Data from the two running tests made during phase 1 are summarized in Table 5. Values provided are mean \pm 1 standard deviation. The second column contains the heart rate taken after the conclusion of the running test (after stage 4). The third column contains the heart rate taken 1 minute later. The fourth column contains the heart rate recovery percentage calculated as described in the equation above. The mean (20.44 for Trial 1 and 20.95 for Trial 2) and standard deviation (5.2 for Trial 1 and 5.6 for Trial 2) were calculated from the sample data. Out of these three measures, $\mathrm{HRr} \%$ had the lowest mean difference, typical error and highest correlation coefficient. When $\mathrm{HRr} \%$ values were averaged, the values on the two days were not significantly different $(p=0.35$, matched t-test).

\begin{tabular}{|l|l|l|l|}
\hline \multicolumn{4}{|c|}{$\begin{array}{c}\text { Table 5. Summary of measurements from the two trials in Phase 1. } \\
\text { Figures are expressed as mean } \pm \text { standard deviation. }\end{array}$} \\
\hline & HR post stage 4 (bpm) & HR 1min post (bpm) & HRr\% \\
\hline Trial 1 & $171 \pm 12.4$ & $136.2 \pm 14.7$ & $20.44 \pm 5.2$ \\
\hline Trial 2 & $164.2 \pm 12.4$ & $129.8 \pm 13.7$ & $20.95 \pm 5.6$ \\
\hline $\begin{array}{l}\text { Difference } \\
\text { (mean } \pm \text { SD) }\end{array}$ & $-6.8 \pm 6.2$ & $-6.40 \pm 5.4$ & $0.51 \pm 2.4$ \\
\hline $\begin{array}{l}\text { 95\% CI } \\
\text { (Difference) }\end{array}$ & -9.72 to 3.9 & -8.93 to 3.9 & -0.60 to 1.6 \\
\hline Typical Error & 4.4 & 3.8 & 1.7 \\
\hline CV\% & 2.7 & 3 & 9.8 \\
\hline Pearson's $\boldsymbol{r}$ & 0.87 & 0.93 & 0.92 \\
\hline
\end{tabular}

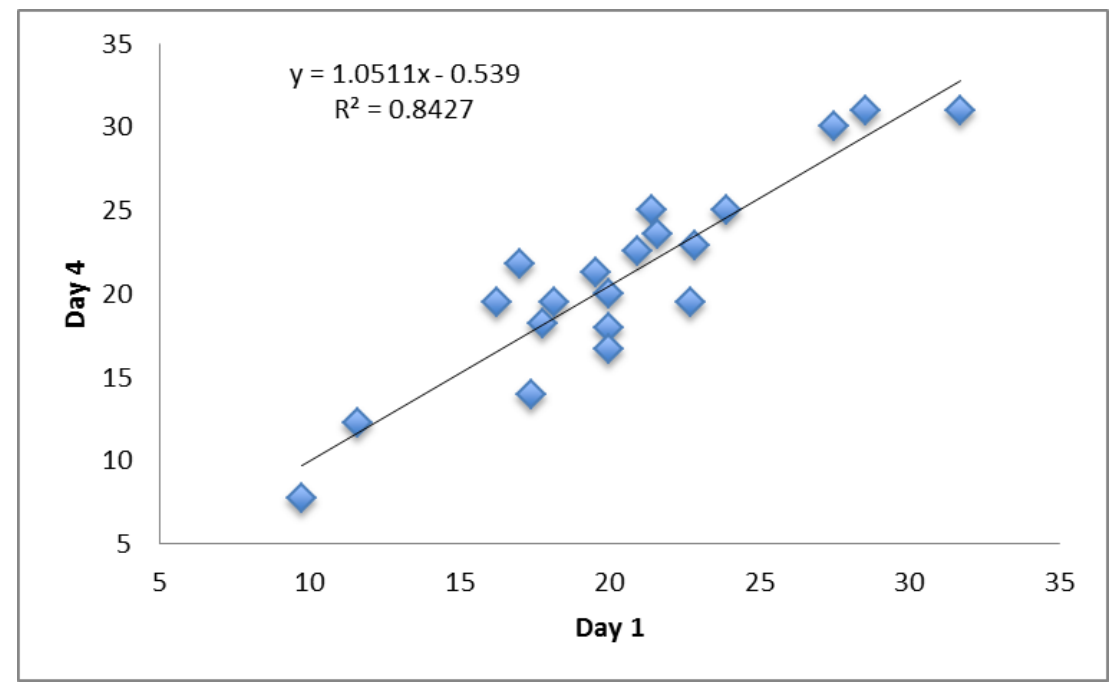

Figure 1. HRr\% plotted for Day 1 and Day 4

A visual comparison between $\mathrm{HRr} \%$ on Day 1 and $\mathrm{HRr} \%$ on Day 4 is shown in Figure 1. This illustrates the correlation between the two measures taken 3 days apart and shows high correlation 
$\left(r^{2}=0.84\right)$. The residuals of the scatterplot were examined graphically and no evidence of bias was detected. Figure 1 suggests that the use of $\mathrm{HRr} \%$ is repeatable given similar testing conditions.

\section{Phase 2: HRr\% in Pre-Season Performance Assessment}

Once the robustness of the $\mathrm{HRr} \%$ measure was established during phase 1 of this research, we applied it to determine if it is correlated to performance, as measured by the $2 \mathrm{~km}$ time trial of athletes during the pre-season training session. In comparison to Phase 1, where the two tests showed very similar results, in Phase 2 there was a marked difference in $\mathrm{HRr} \%$ between week 3 and week 6 , due to the effect of the training protocol. Overall, performance consistently improved over the 12-week training, as measured by the time taken for the $2 \mathrm{~km}$ time trial.

The results of the heart rate taken immediately after stage 4 of the running trial are shown in Figure 2. The heart rate is plotted against the axis on the left, while the time taken to complete the $2 \mathrm{~km}$ run is plotted against the axis on the right. The square or diamond shape indicates the mean, with horizontal bars indicating one standard error. A comparison between weeks 3, 6 and 12 of training shows no significant difference between heart rate. However, results taken from the time trial suggest a decline, indicating increased performance. These results suggest that heart rate is not a satisfactory measure of performance, as it fails to show any change during the training period, although such a change is suggested by the results of the time trial.

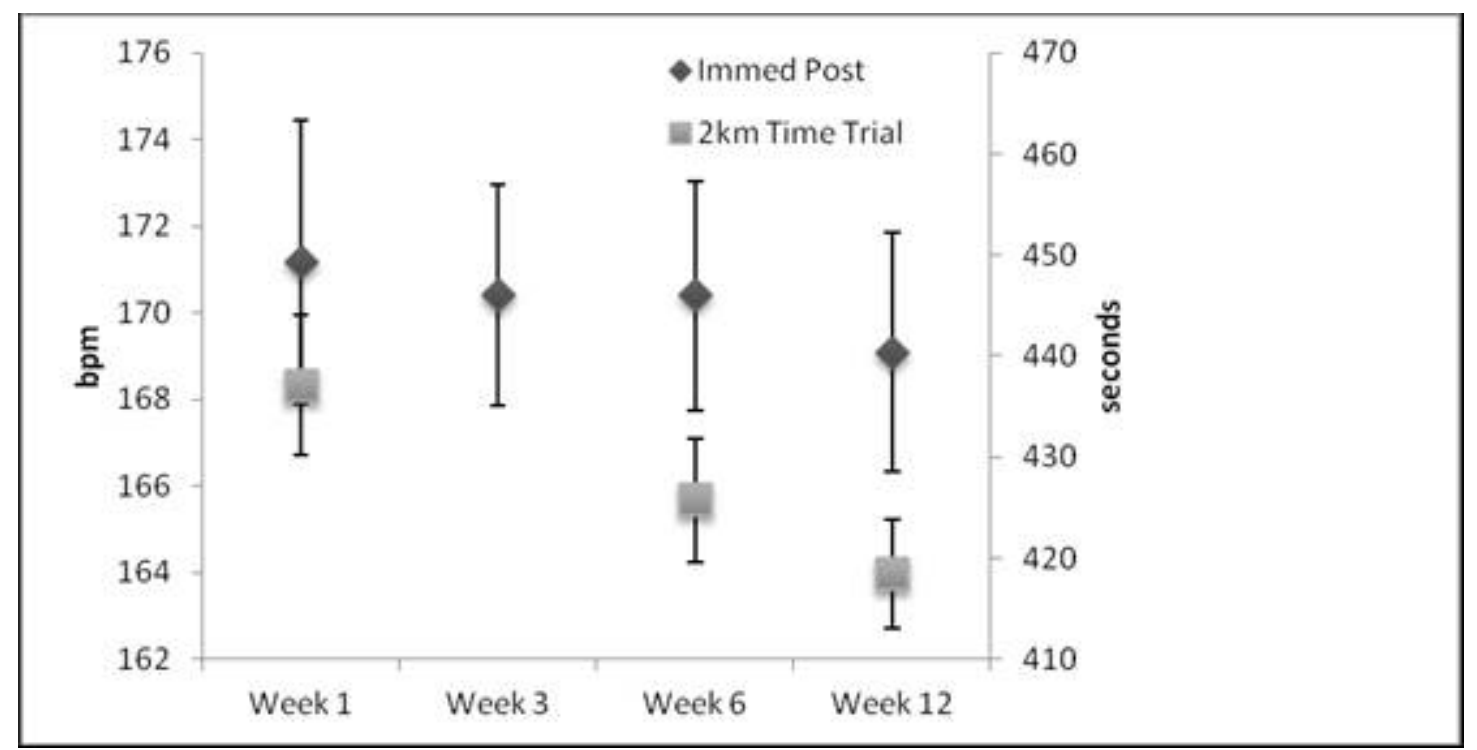

Figure 2. Heart rate and time for $2 \mathrm{~km}$ run measured during pre-season training. Figures are expressed as mean \pm standard error.

The values for $\mathrm{HRr} \%$ are shown in Figure 3, where they are plotted against the axis on the left. As in Figure 2, the time taken for the $2 \mathrm{~km}$ run is plotted against the axis on the right. The square or diamond shape indicates the mean, with horizontal bars indicating one standard error. $\mathrm{HRr} \%$ was significantly different in weeks 6 and 12 ( $\mathrm{p}<0.001$ and $\mathrm{p}<0.05$ respectively) compared to week 1 . The Cohen effect size for week 1 compared to weeks 3,6 , and 12 was $-0.03,0.92$, and 0.46 respectively. $\mathrm{HRr} \%$ for week 3 compared to week 6 and 12 was statistically significant at $\mathrm{p}<0.0001$ and $\mathrm{p}<0.005$ respectively. The Cohen effect size from week 3 to week 6 and 12 was 0.96 and 0.49 respectively. Results were also significantly different between week 6 and week 12 $(p<0.05)$ with a Cohen effect size of -0.46 . 


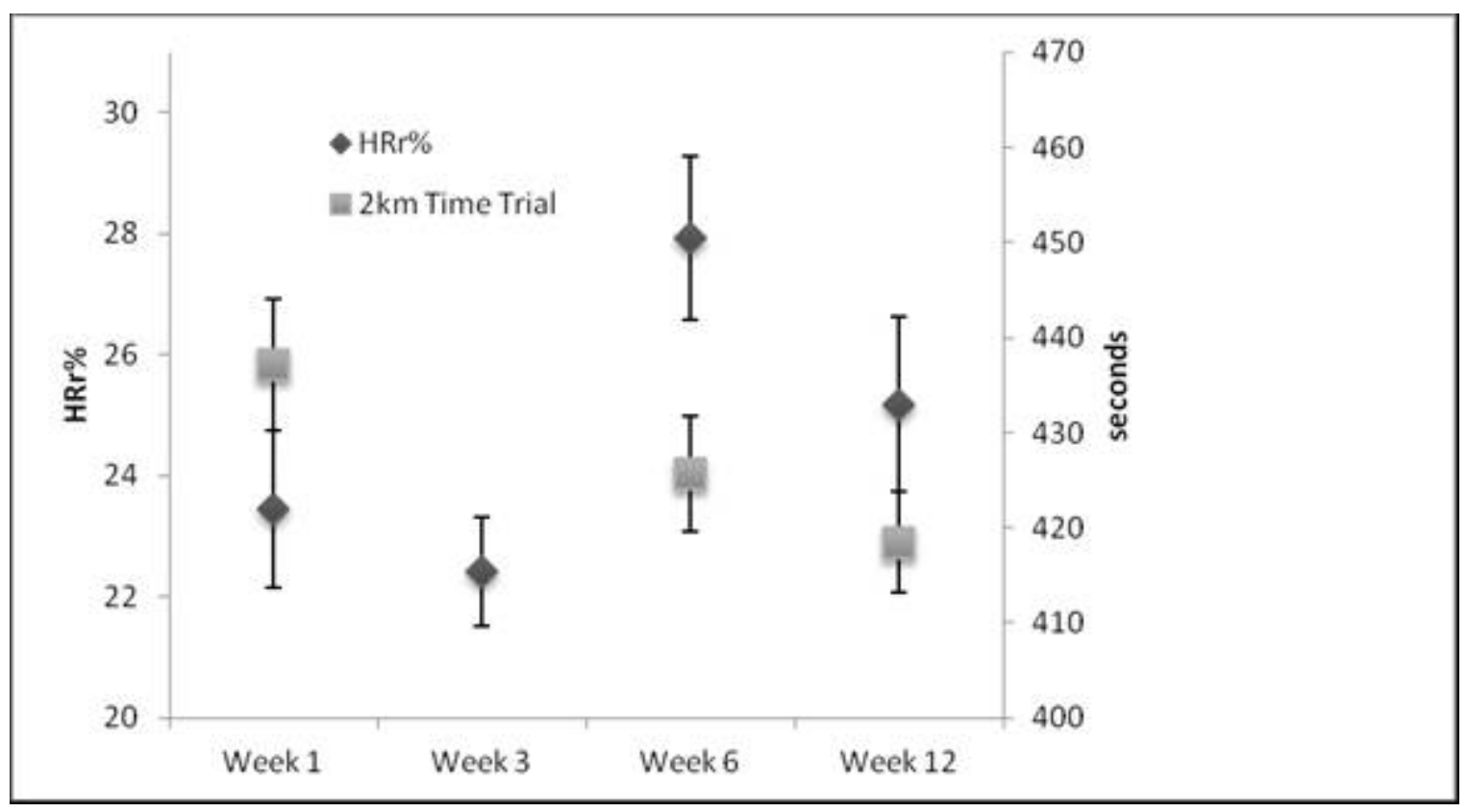

Figure 3. Time trial and $\mathrm{HRr} \%$ results measured during pre-season training.

Figures are expressed as mean \pm standard error.

$\mathrm{HRr} \%$ is related to aerobic fitness but is also sensitive to exercise load. As overall fitness improved $\mathrm{HRr} \%$ also improved, but superimposed on this trend were fluctuations related to the exercise load in the immediately preceding training period. Investigation of performance using the $2 \mathrm{~km}$ time trial test indicated that there was a steady improvement in performance over the 12 week period with an increase of speed over the 2-kilometre distance of $11 \mathrm{sec}(2.54 \%)$ between Week 1 and Week 6, and a final improvement of $4.6 \%$ at week 12 . $\mathrm{HRr} \%$ showed sensitivity to training load with a significant difference between the test days using Kruskal-Wallis statistics ( $\mathrm{p}=0.016$ ) and a significant increase in $\mathrm{HRr} \%$ following the lower training load at Week 6.

\section{Discussion}

Team sport athletes are required to undertake a pre-season training program each year that is designed to increase fitness and optimally prepare them for the upcoming competition season. The pre-season training is designed to progressively increase load to improve fitness. This progressive increase in load requires a continual adjustment to maintain a balance between a high training load and adequate recovery (Busso, 2003). The balance needs to be monitored to create the optimum application of the training load. If it is unbalanced in favour of high load, then training cannot be tolerated and symptoms of fatigue will develop. A continuous imbalance in the loadrecovery relationship will lead to either 'functional' or 'non-functional' over-reaching. In the long term this can develop into overtraining syndrome with detrimental effects on performance and health, indicated by a decrease in heart rate recovery in the case of over-training (Baumert et al., 2006; Borresen \& Lambert, 2008; Meeusen et al., 2006). However a program that is too high in recovery will not increase fitness, as the stimulus is not sufficient enough to create a positive change in homeostasis. This is indicated by either a plateau or increase in the submaximal heart rate (Zavorsky, 2000).

While performance can be influenced by frequency, intensity, volume and duration of training load, exercise recovery is influenced by less controllable factors such as stress, sleeping quality, nutrition and psychosocial factors (Borresen \& Lambert, 2008; Jeukendrup, 2002; Kentta \& 
Hassmen, 1998). To be able to adapt training loads based on the measurements during the warmup requires confidence in the results.

\section{Modified HIMS Test}

During phase 1 of our study to determine the suitability of $\mathrm{HRr} \%$ as a performance indicator and utilising a modified assessment protocol, we indicated that heart rate at the end of stage 4 varied the least. However both heart rate at the end of stage 4 and 1-minute post exercise had sufficient precision to detect changes when the HR differed by as low as $9 \mathrm{bpm}$.

The $\mathrm{HRr} \%$ test as performed here is designed to determine whether athletes require a change in training load to maintain optimum body homeostasis during the warm-up of training. Changes to training loads can be applied immediately if the parameters of maintaining homeostasis have not been met. Future research should examine whether these markers are sensitive for tracking changes over a longer period and also involve the use of heart rate monitors.

The current modification made to the HIMS test provides an appropriate workload for each athlete. The modification of the HIMS test is intended to address the different style of sport Australian football players play and to provide the type of test that most accurately reflects the game conditions for assessing aerobic fitness and performance. In addition, the initial HIMS, being designed for a different sport, also stresses the groin area as it requires repeated sudden change of direction. Our modification is a reliable measure for monitoring changes in heart rate and subsequent recovery rates. The current test measures the fitness levels of professional team sport athletes based on the assumption that increasing and decreasing aerobic fitness corresponds with the decrease and increase in heart rate respectively at submaximal exercise intensities. The heart rate recovery percentage is a good indicator of disturbance to homeostasis as a result of accumulation of fatigue or functional over-reaching (Lamberts et al., 2009).

\section{HRr\% Assessment During a 12-Week Preseason Training}

Over the 12-week training period the training load was varied to assess the effect of acute training load on $\mathrm{HRr} \%$ and performance in the same controlled cohort. Previous studies have shown that heart rate may remain stable over fluctuations in training load with $\mathrm{HRr} \%$ being more sensitive to training conditions (Buchheit \& Gindre, 2006). Performance continued to improve significantly regardless of training load. This continued improvement in performance combined with an improvement associated with $\mathrm{HRr} \%$ suggests that acute changes in training load can be beneficial either over an extended training period as was the case in our study of 12 weeks or implementing training load adjustments in accordance with individual athlete requirements.

In the current study we found that an increase in $\mathrm{HRr} \%$ after 6-weeks corresponded with an increase in aerobic fitness as measured through a $2 \mathrm{~km}$ time trial. This increase in fitness accompanied by an increase in $\mathrm{HRr} \%$ over a 6 -week period agrees with the findings of several previous studies (Buchheit et al., 2010; Lamberts et al., 2009; Sugawara et al., 2001). The heart rate recovery remained significantly higher than week 1 after the completed 12-week period, although it decreased when compared to week 6. This supports the findings of Buchheit and Gindre (2006) who found HRr to be a good indicator of training load. The data clearly show the effect of training load, with the decreased training load from week 4 and 5 inclusive resulting in an improved heart rate recovery percentage and thus a lesser risk of over-training, yet still improving performance. Undertaking the greater training load again in weeks 6 to 12 reduced the $\mathrm{HRr} \%$ but the athletes retained the improvement in performance, which was steady throughout the trial. In comparing the changes from week 3 to 6 , there is a decrease in training load over weeks 4 and 5 and a corresponding drop of 1-minute post heart rate in week 6 which contributes to the change in $\mathrm{HRr} \%$ at week 6 . This increase in vagal activation can be attributed to both an increase in aerobic 
fitness and decrease in training load (Sugawara et al., 2001). Similar to the findings of Borresen and Lambert (2008), HRr\% decreased with increases in training load between week 6 and week 12. Sub-maximal HR (Figure 2) was not affected following an acute change in training load at week 6 . Heart rate during exercise measures cardiac load, whereas HRr may reflect autonomic nervous system function and indicate the body's capacity to respond to exercise (Borresen \& Lambert, 2007). The finding of a non-significant change in $\mathrm{HRr} \%$ over the first 3 -week period could be a combination of an increase in aerobic fitness along with increase in training load in agreement with previous findings (Buchheit \& Gindre, 2006).

\section{Conclusion}

This is the first study to have focused specifically on the reliability of measuring submaximal heart rate during a fitness program in professional team sport athletes. During the test, the exercise intensity was controlled and normal activities of daily living were maintained. This study was designed to identify the variation of heart rate within the normal day-to-day operation of a pre-season training schedule, so that interpretation of submaximal heart rate changes and the associated parameters may be interpreted with greater clarity, and identification of changes in homeostasis can be interpreted more precisely.

The main finding of the first phase of the study was that $\mathrm{HRr} \%$ was reliable $(\mathrm{R}=0.92)$ and valid for the assessment of training load with low intra-individual variation, as required for a robust test (Hopkins, 2002). The practical application of this result is that the modified HIMS test has sufficient precision to detect significant changes in $\mathrm{HRr} \%$ when the $\mathrm{HRr} \%$ changes by at least $1.62 \%$. Based on the observation that the heart rate recovers faster with increasing aerobic fitness (Dennis $\&$ Noakes, 1988), the heart rate recovery percentage increases with increasing fitness and therefore provides an index of training effectiveness.

The main finding of the second phase was that the effect of decreased training load from weeks 4 and 5 inclusive resulted in an improved heart rate recovery percentage and thus a lesser risk of over-training. We propose that it is the combination of aerobic fitness and training load status that is reflected in the $\mathrm{HRr} \%$. $\mathrm{HRr} \%$ is therefore a good indicator of performance throughout a competition cycle.

We agree with Buchheit and Gindre (2006) that training load influences heart rate recovery parameters. Our finding that the improvement of aerobic fitness contributes to an increase in $\mathrm{HRr} \%$ also agrees with the findings of Lamberts and colleagues (2009).

\section{Ethics Declaration}

The study was approved by The Australian Institute of Sport Ethics and Research Committee and all participants gave informed consent in writing.

\section{Conflict of Interest}

The authors declare that they have no conflict of interest. 


\section{References}

Aziz, A. R., Kilding, A. E., \& Teh, K. C. (2006). Heart rate recovery post-maximal exhaustive exercise and its correlation with maximal aerobic power in trained team sport athletes. Journal of Exercise Science \& Fitness, 4(2), 110-116.

Barbeau, P., Serresse, O., \& Boulay, M. R. (1993). Using maximal and submaximal aerobic variables to monitor elite cyclists during a season. Medicine \& Science in Sports \& Exercise, 25, 1062-1069.

Baumert, M., Brechtel, L. M., Lock, J., Voss, A., \& Abbott, D. (2006). Scaling graphs of heart rate time series in athletes demonstrating the VLF, LF and HF regions. Physiological Measurements, 27, N35N39. doi:10.1088/0967-3334/27/9/N1

Borresen J., \& Lambert, M. I. (2007). Changes in heart rate recovery on response to acute changes in training load. European Journal of Applied Physiology, 101, 503-511.

Borresen, J., \& Lambert, M. I. (2008). Autonomic control of heart rate during and after exercise. Sports Medicine, 38, 633-646.

Bosquet, L., Gamelin, F.X., \& Berthoin, S. (2008). Reliability of postexercise heart rate recovery. International Journal of Sports Medicine, 29, 238-243.

Buchheit, M., Duche, P., Laursen, P. B., \& Ratel, S. (2010). Postexercise heart rate recovery in children: relationship with power output, blood pH, and lactate. Applied Physiology, Nutrition, \& Metabolism, 35(2), 142-150. doi: 110.1139/H1109-1140

Buchheit, M., \& Gindre, C. (2006). Cardiac parasympathetic regulation: respective associations with cardiorespiratory fitness and training load. American Journal of Physiology - Heart \& Circulatory Physiology, 291(1), H451-H458. doi:10.1152/ajpheart.00008.2006

Buchheit, M., Millet, G. P., Parisy, A., Pourchez, S., Laursen, P. B., \&Ahmaidi, S. (2008). Supramaximal training and post-exercise parasympathetic reactivation in adolescents. Medicine \& Science in Sports \& Exercise, 40, 362-371.

Buchheit, M., Papelier, Y., Laursen, P. B., \&Ahmaidi, S. (2007). Noninvasive assessment of cardiac parasympathetic function: post-exercise heart rate recovery or heart rate variability? American Journal of Physiology: Heart \& Circulation Physiology, 293, H8-H10.

Buchheit, M., Simpson, M. B., Al Haddad, H., Bourdon, P. C., \& Mendez-Villanueva, A. (2012). Monitoring changes in physical performance with heart rate measures in young soccer players. European Journal of Applied Physiology, 112(2), 711-723. doi:10.1007/s00421-011-2014-0.

Busso, T. (2003). Variable dose-response relationship between exercise training and performance. Medicine \& Science in Sports \& Exercise, 35(7), 1188-1195.

Chatterjee, S., Chatterjee, P., \& Bandyopadhyay, A. (2005). Validity of Queen's College step test for estimation of maximum oxygen uptake in female students. Indian Journal of Medical Research, 121, 3235 .

Cohen, J. (1988). Statistical power analysis for the behavioral sciences (2nd ed.). Hillsdale, NJ: Lawrence Earlbaum Associates.

Coutts, A. J., Reaburn, P., Piva, T. J., \& Rowsell, G. J. (2007). Monitoring for overreaching in rugby league players. European Journal of Applied Physiology, 99(3), 313-324.

Dellal, A., Keller, D., Carling, C., Chaouachi, A., Wong, D., \&Chamari, K. (2010). Physiologic effects of directional changes in intermittent exercise in soccer players. Journal of Strength \& Conditioning Research, 24(12), 3219-3226.

Dennis, S. C., \&Noakes, T. D. (1988). Physiological and metabolic responses to increasing work rate: Relevance for exercise prescription. Journal of Sports Science, 16, S77-S84. 
Edwards, A. M., MacFayden, A. M., \& Clark N. (2003). Test performance indicators from a single soccer specific test differentiate between highly trained and recreationally active soccer players. Journal of Sports Med Phys Fitness, 43, 14-20.

Hoffman, J. R., Epstein, S., Einbinder, M., \& Weinstein, Y. (1999). The influence of aerobic capacity on anaerobic performance and recovery indices in basketball players. Journal of Strength \& Conditioning Research, 13, 407-411.

Hopkins, W. G. (2002). A new view of statistics: A scale of magnitudes for effect statistics. Retrieved December 26, 2012, from http://www.sportsci.org/resource/stats/effectmag.html

Hopkins, W. G., \&Hewson, D. J. (2001). Variability of competitive performance of distance runners. Medicine \& Science in Sports \& Exercise, 33, 1588-1592.

Hottenrott, K., Hoos, O., \& Esperer, H. (2006). Heart rate variability and physical exercise. Herz, 31(6), 544-552. doi:10.1007/s00059-006-2855-1

Javorka, M., Zila, I., Balharek, T., \& Javorka, K. (2002). Heart rate recovery after exercise: Relations to heart rate variability and complexity. Brazilian Journal of Medical \& Biological Research, 35(8), 9911000 .

Jeukendrup, A. E. (2002). Regulation of fat metabolism in skeletal muscle. Annals of the New York Academy of Science, 967, 217-235.

Kannankeril, P. J., Le, F. K., Kadish, A. H., \& Goldberger, J. J. (2004). Parasympathetic effects on heart rate recovery after exercise. Journal of Investigative Medicine, 52(6), 394-401.

Kenttä, G., \& Hassmen, P. (1998). Overtraining and recovery. Sports Medicine, 26(1), 1-16.

Kiviniemi, A., Hautala, A. J., Seppänen, T., Mäkikallio, T. H., Huikuri, H. V., \&Tulppo, M. (2004). Saturation of high-frequency oscillations of R-R intervals in healthy subjects and patients after acute myocardial infarction during ambulatory conditions. American Journal of Heart and Circulation Physiology, 287, H1921-H1927.

Kraemer, W. J., Torine, J. C., Silvestre, R., French, D. N., Ratamess, N. A., Spiering, B. A., Hatfield, D. L., Vingren J. L., \&Volek, J. S. (2005). Body size and composition of National Football League players. Journal of Strength and Conditioning Research, 19(3), 485-489.

Kreider, R. B., Fry, A., \& O’Toole, M. L. (1998). Overtraining in sport. Champaign, IL; Human Kinetics.

Lamberts, R. P., Lemmink, K. A. P. M., Durandt, J. J., \& Lambert, M. I. (2004). Variation in heart rate during submaximal exercise: Implications for monitoring training. Journal of Strength and Conditioning Research, 18, 641-645.

Lamberts, R. P., Maskell, S., Borresen, J., \& Lambert, M. I. (2011). Adapting workload improves the measurement of heart rate recovery. International Journal of Sports Medicine, 32(9), 698-702. doi:10.1055/s-0031-1275357

Lamberts, R. P., Rietjens, G. J., Tijdink, H. H., Noakes, T. D., \& Lambert,M. I. (2010). Measuring submaximal performance parameters to monitor fatigue and predict cycling performance: A case study of a world-class cyclo-cross cyclist. European Journal of Applied Physiology, 108(1), 183-190. doi:10.1007/s00421-009-1291-3

Lamberts, R. P., Swart, J., Capostagno, B., Noakes, T. D., \& Lambert, M. I. (2009). Heart rate recovery as a guide to monitor fatigue and predict changes in performance parameters. Scandinavian Journal of Medicine \& Science in Sports, 20(3), 449-457.

Martinmäki, K., \&Rusko, H. (2007). Time-frequency analysis of heart rate variability during immediate recovery from low and high intensity exercise. European Journal of Applied Physiology, 102, 353-360.

McNair, D., Lorr, M., \& Droppleman, L. (1971). Profile of mood states. San Diego, California: Educational and Industrial Testing Service. 
Meeusen, R., Watson, P., Hasegawa, H., Roelands, B., \& Piacentini, M. F. (2006). Central fatigue: The serotonin hypothesis and beyond. Sports Medicine, 36(10), 881-909.

Reilly, T., Robinson, G., \& Minors, O. S. (1984). Some circulatory responses to exercise at different times of day. Medicine \& Science in Sports \& Exercise, 16, 477-482.

Rushall, B. S. (1990). A tool for measuring stress tolerance in elite athletes. JASP, 2(1), 51-66.

Savin, W. M., Davidson, D. M., \& Haskell, W. L. (1982). Autonomic contribution to heart rate recovery from exercise in humans. Journal of Applied Physiology, 53(6), 1572-1575.

Scharhag-Rosenberger, F., Meyer, T., Walitzek, S., \& Kindermann, W. (2009). Time course of changes in endurance capacity: A 1-yr training study. Medicine \& Science in Sports \& Exercise, 41, 1130-1137.

Sugawara J., Murakami H., Maed S., Kuno S., \& Matsuda, M. (2001). Change in post-exercise vagal reactivation with exercise training and detraining in young men. European Journal of Applied Physiology, $85,259-263$.

Wang, N. C., Chicos, A., Banthia, S., Bergner, D. W., Lahiri, M. K., Ng, J., Subafçius, H., Kadish, A. H., \& Goldberger, J. J. (2011). Persistent sympathoexcitation long after submaximal exercise in subjects with and without coronary artery disease. American Journal of Physiology - Heart and Circulatory Physiology, 301(3), H912-H920. doi:10.1152/ajpheart.00148.2011

Zavorsky, G. S. (2000). Evidence and possible mechanisms of altered maximum heart rate with endurance training and tapering. Sports Medicine, 29, 13-26.

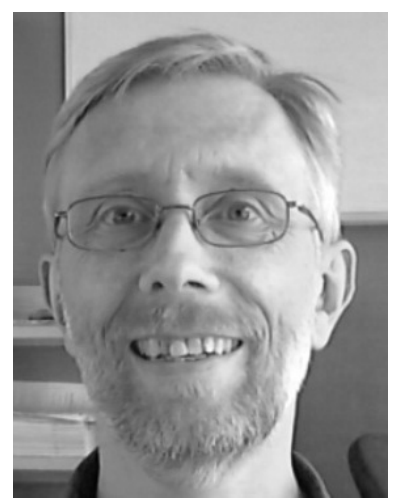

\section{Biographies}

David Cornforth holds the B.Sc. in Electrical and Electronic Engineering from Nottingham Trent University, UK (1982), and the Ph.D. in Computer Science from the University of Nottingham, UK (1994). $\mathrm{He}$ has been an educator and researcher at Charles Sturt University, the University of New South Wales, and currently at the University of Newcastle. He has also been a research scientist at the Commonwealth Scientific and Industrial Research Organisation (CSIRO), Newcastle, Australia. His research interests are in health information systems, pattern recognition, artificial intelligence, multi-agent simulation, and optimization. He is convenor of the Applied Informatics Research Group, University of Newcastle.

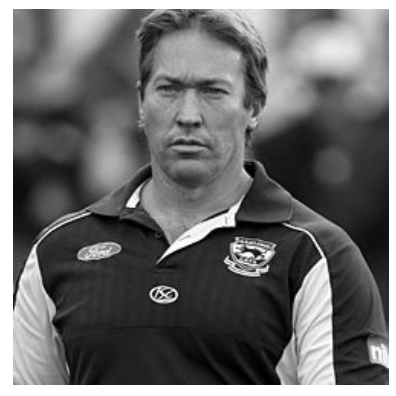

Dean Robinson is a high performance fitness coach for Australian Rules Football. He joined the Geelong, Victoria, club in 2007 where he was the conditioning coach. He joined the Gold Coast club in 2010 then joined Essendon before the 2012 pre-season and oversaw the players' strength and conditioning program for that year. 


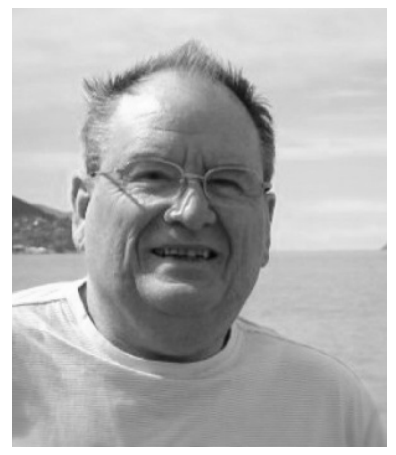

Ian Spence holds a B.Sc.(Hons.) in biophysics from the University of Sydney and Ph.D. in physiology from the Monash University (1975). Following a period working in the pharmaceutical industry he has been involved as a teacher and researcher in pharmacology and toxicology at the University of Sydney where he was Head of the Discipline of Pharmacology from 2004-2009. He is currently Associate Dean (International) in the Faculty of Science at the University of Sydney.

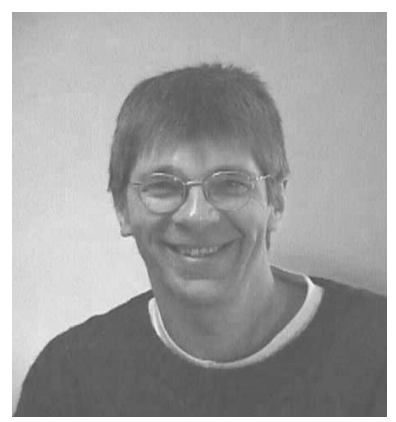

Herbert Jelinek holds the B.Sc. (Hons.) in human genetics from the University of New South Wales, Australia (1984), Graduate Diploma in neuroscience from the Australian National University (1986) and $\mathrm{Ph}$.D. in medicine from the University of Sydney (1996). He is Clinical Associate Professor with the Australian School of Advanced Medicine, Macquarie University, and a member of the Centre for Research in Complex Systems, Charles Sturt University, Australia. Dr Jelinek is currently visiting Associate Professor at Khalifa University of Science, Technology and Research, Abu Dhabi, UAE. He is a member of the IEEE Biomedical Engineering Society and the Australian Diabetes Association. 